\title{
A hybrid method for the design of oversampled uniform DFT filter banks
}

\author{
Ka Fai Cedric Yiu ${ }^{\mathrm{a}, *}$, Nedelko Grbićc ${ }^{\mathrm{b}}$, Sven Nordholm ${ }^{\mathrm{c}, 1}$, Kok Lay Teo ${ }^{\mathrm{d}}$ \\ ${ }^{a}$ Department of Industrial and Manufacturing Systems Engineering, The University of Hong Kong, Pokfulam Road, Hong Kong \\ ${ }^{\mathrm{b}}$ Department of Signal Processing, Blekinge Institute of Technology, SE-372 25 Ronneby, Sweden \\ ${ }^{\mathrm{c}}$ WATRI, Western Australian Telecommunications Research Institute, Australia \\ ${ }^{\mathrm{d}}$ Department of Mathematics and Statistics, Curtin University of Technology, Perth, WA 6845 Australia
}

Received 27 January 2003; received in revised form 15 June 2004

Available online 28 September 2005

\begin{abstract}
Subband adaptive filters have been proposed to speed up the convergence and to lower the computational complexity of time domain adaptive filters. However, subband processing causes signal degradations due to aliasing effects and amplitude distortions. This problem is unavoidable due to further filtering operations in subbands. In this paper, the problem of aliasing effect and amplitude distortion is studied. The prototype filter design problem is formulated as a multicriteria optimization problem and all the Pareto optima are sought. Since the problem is highly nonlinear and nonsmooth, a new hybrid optimization method is proposed. Different prototype filters are used and their performances are compared. Moreover, the effect of the number of subbands, the oversampling factors and the length of prototype filter are also studied. We find that prototype filters designed via Kaiser or Dolph-Chebyshev window provide the best overall performance. Also, there is a critical oversampling factor beyond which the improvement in performance is not justified. Finally, if the length of the prototype filter increases with the number of subbands, an increase in the subband level will not deteriorate the performance.
\end{abstract}

(C) 2005 Elsevier B.V. All rights reserved.

Keywords: Subband adaptive filter; Aliasing effect; Amplitude distortion; Simulated annealing; Pareto optimum

\section{Introduction}

Adaptive filtering in subbands is an attractive alternative to the full-band scheme in many applications to achieve faster convergence and lower computational cost. In a typical subband

\footnotetext{
*Corresponding author. Tel.: + 85222415956 ; fax: +85228586535 .

E-mail address: cedric@hkucc.hku.hk (K.F.C. Yiu).

${ }^{1}$ A joint venture between The University of Western Australia and Curtin University of Technology, Perth, Australia
}

adaptive filter, the filter input is first partitioned into a set of subband signals through an analysis filter bank. These subband signals are then decimated to a lower rate and passed through a set of independent or partially independent adaptive filters that operate at the decimated rate. The outputs from these filters are subsequently combined using a synthesis filter bank to reconstruct the full-band output. The DFT multirate filter banks are commonly used for efficient realization of the analysis and synthesis filter banks $[1,2]$. 
However, the analysis of a signal into a subband representation and the synthesis back into its original full-band form has several difficulties. Noticeably, subband filtering introduces signal degradations which include signal distortions and aliasing effects [3]. It is well known that a filter bank can be designed alias-free and perfectly reconstructed when certain conditions are met by the analysis and synthesis filters. However, any filtering operation in the subbands may cause a possible phase and amplitude change and thereby altering the perfect reconstruction property. There are tradeoffs in controlling both the aliasing effect and the distortion level. Non-critical decimation has been suggested in [4] to improve the overall performance of the filter banks. Depending on the level of oversampling, the cost of computation also increases significantly.

In general, the filter bank design problem is a multi-criteria decision problem, where the criteria are the level of distortion and the level of aliasing effect. A very sharp prototype filter will decrease the aliasing effect and distortion, but the length of the filter is usually prohibitively long. Depending on the computation complexity, the length of the prototype filter is usually limited. Within this limit, the optimal filter is sought. If a simple least-squares technique is used to minimize both criteria together, there is no direct control over each individual criterion. During the design process, it is therefore not possible to specify the performance of the filter bank in advance. Methods have been proposed to minimize both criteria simultaneously, such as [5]. However, more flexible design of the prototype filter has not been considered, and individual criterion is not controlled directly.

The performance of the filter bank depends on the choice of prototype filter, the length of it, the number of subbands, and the oversampling factor. Here, we study the optimal designs for different combinations of parameters. A multi-criteria formulation is employed to trade off the aliasing effect against the distortion level. In order to allow for the worst scenario, the maximum norm is applied. Consequently, the filter design problem becomes highly nonlinear and both the cost function and constraint are not differentiable. In order to tackle this problem, the $L_{1}$ exact penalty function is first applied to transform the constrained problem into an equivalent unconstrained problem. A new hybrid method is then proposed to solve the resultant highly nonlinear optimization problem. The hybrid method combines the simulated annealing (which has the advantage of escaping from local minima) and the simplex search method (which is a noderivative search method to locate local minima) to achieve fast convergence to the global minimum. One main desirable property of the proposed hybrid descent method is that the convergence is monotonic.

This approach is versatile in the way that a specific performance of the filter bank can be imposed in advance. The aliasing and distortion level can be controlled easily and the corresponding optimal weights can be found. In this way, all the Pareto optima can be sought. In assessing the performance, different prototype filter designs are studied. These include the window method with the Hamming window, Kaiser window and DolphChebyshev window, and the minimax method. We show that Kaiser and Dolph-Chebyshev window give the best overall performance with or without oversampling. Finally, the effects of the oversampling factor, the number of subbands and the length of the prototype filter are investigated.

\section{The uniform DFT modulated filter bank}

In a typical analysis-synthesis DFT filter bank, two sets of filters form a uniform DFT analysis filter bank and synthesis filter bank. Assume the same prototype filter is applied for both analysis and synthesis, the subband filters are related to the prototype filter, $h_{0}(n)$, by means of modulation as

$$
\begin{aligned}
H_{k}(z) & =H_{0}\left(z W_{K}^{k}\right)=\sum_{n=-\infty}^{\infty} h_{0}(n)\left(z W_{K}^{k}\right)^{-n} \\
& =\boldsymbol{h}^{\mathrm{T}} \phi\left(z W_{K}^{k}\right), \quad k=0, \ldots, K-1,
\end{aligned}
$$

where $W_{K}=\mathrm{e}^{-\mathrm{j} 2 \pi / K}, \boldsymbol{h}=[h(0), \ldots, h(L-1)]^{\mathrm{T}}$ and $\phi(z)=\left[1, z^{-1}, \ldots, z^{-(L-1)}\right]^{\mathrm{T}}$. Each subband signal is decimated by a factor $D$. An implementation of such a filter bank is depicted in Fig. 1. A typical analysis operation can be summarized in Fig. 2 . Using the subband signal definitions according to Fig. 1, we can describe the signal path through the filter-bank realization. Each branch signal, $V_{k}(z)$, is simply a filtered input signal defined as

$$
\begin{aligned}
& V_{k}(z)=H_{k}(z) X(z)=H_{0}\left(z W_{K}^{k}\right), X(z), \\
& k=0, \ldots, K-1 .
\end{aligned}
$$

The decimators cause a summation of repeated and expanded spectrum of the input signal 


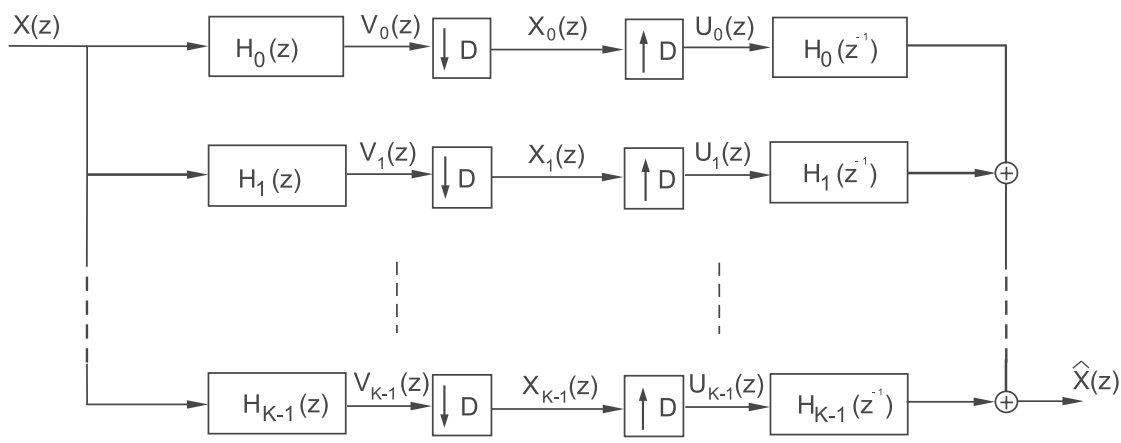

Fig. 1. Direct form realization of an analysis and synthesis filter-bank.

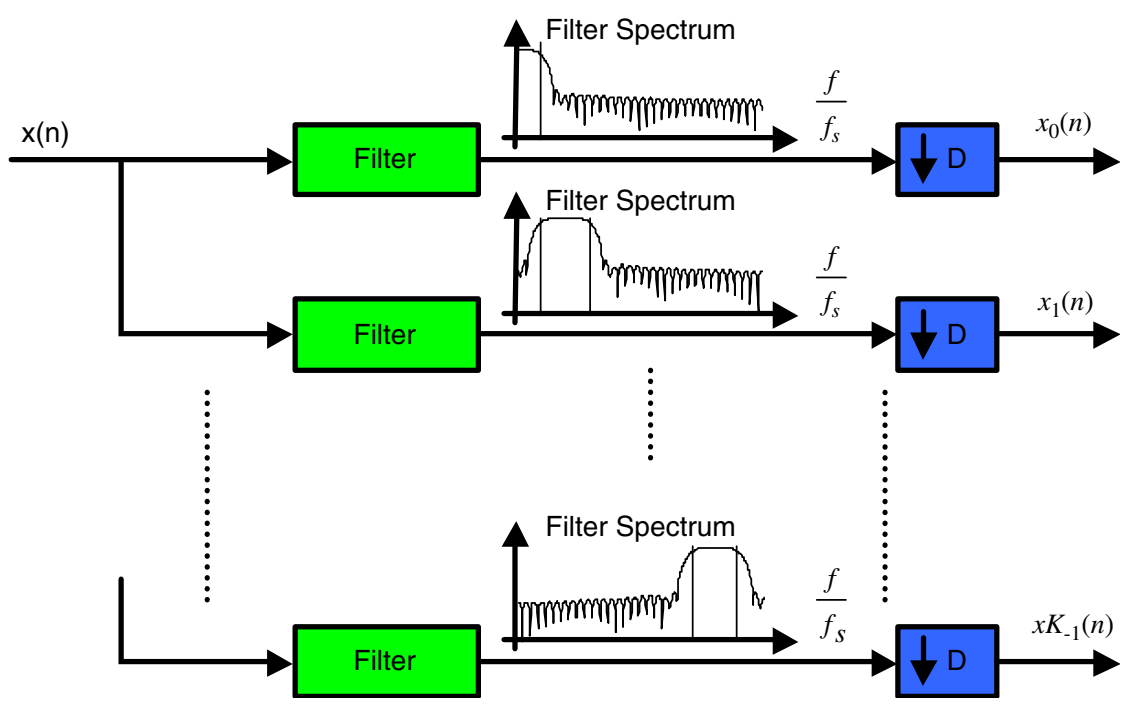

Fig. 2. A typical analysis operation.

according to

$$
\begin{aligned}
X_{k}(z)= & \frac{1}{D} \sum_{l=0}^{D-1} V_{k}\left(z^{1 / D} W_{D}^{l}\right) \\
= & \frac{1}{D} \sum_{l=0}^{D-1} H_{0}\left(z^{1 / D} W_{K}^{k} W_{D}^{l}\right) X\left(z^{1 / D} W_{D}^{l}\right), \\
& k=0, \ldots, K-1,
\end{aligned}
$$

where $W_{D}=\mathrm{e}^{-\mathrm{j} 2 \pi / D}$. The interpolators have a compressing effect according to

$$
\begin{aligned}
& U_{k}(z)=X_{k}\left(z^{D}\right)=\frac{1}{D} \sum_{l=0}^{D-1} H_{0}\left(z W_{K}^{k} W_{D}^{l}\right) X\left(z W_{D}^{l}\right), \\
& k=0, \ldots, K-1 .
\end{aligned}
$$

Finally, the signal will be synthesized by the reconstruction filters and we can state input-output relationship as

$$
\begin{aligned}
\widehat{X}(z) & =\sum_{k=0}^{K-1} F_{k}(z) U_{k}(z) \\
& =\frac{1}{D} \sum_{l=0}^{D-1} X\left(z W_{D}^{l}\right) \sum_{k=0}^{K-1} H_{0}\left(z W_{K}^{k} W_{D}^{l}\right) H_{0}^{*}\left(z W_{K}^{k}\right),
\end{aligned}
$$

where the superscript * denotes the conjugate. This expression can be rewritten as

$\widehat{X}(z)=\sum_{l=0}^{D-1} A_{l}(z) X\left(z W_{D}^{l}\right)$,

where

$$
A_{l}(z)=\frac{1}{D} \sum_{k=0}^{K-1} H_{0}\left(z W_{K}^{k} W_{D}^{l}\right) H_{0}^{*}\left(z W_{K}^{k}\right) .
$$


If $A_{l}(z)=0$ for $l=1,2, \ldots, D-1$, and $A_{0}(z)=$ $a z^{-b}$, for any $a, b$ where $a \neq 0$, we get a perfect reconstruction filter-bank. However, any filtering operation in the subbands may cause a possible phase and amplitude change and thereby altering the perfect reconstruction property. Our main objective is to find the prototype filter coefficients $\boldsymbol{h}$ to minimize both the aliasing power and the amplitude distortion defined as

$A_{P}=\max _{\omega}\left(\sum_{l=1}^{D-1}\left|A_{l}\left(\mathrm{e}^{-\mathrm{j} \omega}\right)\right|\right)$,

$A_{D}=\max _{\omega}\left(1-\left|A_{0}\left(\mathrm{e}^{-\mathrm{j} \omega}\right)\right|\right)$.

The aliasing effect is best understood by Figs. 3 and 4 where a critical sampling clearly create severe aliasing effect due to the transition region of the prototype filter. When the oversampling increases, the lines of aliasing will gradually move further to reduce the aliasing effect. In optimizing the prototype filter, simply minimizing a sum of both measures may result in performance skewing toward one extreme. There is no easy way to introduce any scaling factor to adjust such uneven performances. Because there are more than one objective in the design of the filter-bank, it is basically a multi-criteria design problem [6,7]. When different scaling factors are applied to the criteria in the design process, a solution set can be derived in which all solutions are efficient, or Pareto optima. In the present context, the set of weights $\boldsymbol{h}^{\prime}$ is a Pareto optimum if and only if there does not exist a set of weights $\boldsymbol{h}$ such that

$$
A_{P}(\boldsymbol{h}) \leqslant A_{P}\left(\boldsymbol{h}^{\prime}\right), \quad A_{D}(\boldsymbol{h}) \leqslant A_{D}\left(\boldsymbol{h}^{\prime}\right)
$$

with strict inequality to at least one of the criteria. In order to solve for the Pareto optima, one of the criteria can be formulated as a constraint instead so that it becomes a nonlinear programming problem. An additional advantage of using this formulation is that the constraint can be adjusted freely to select the desired filter from the set of Pareto optima.

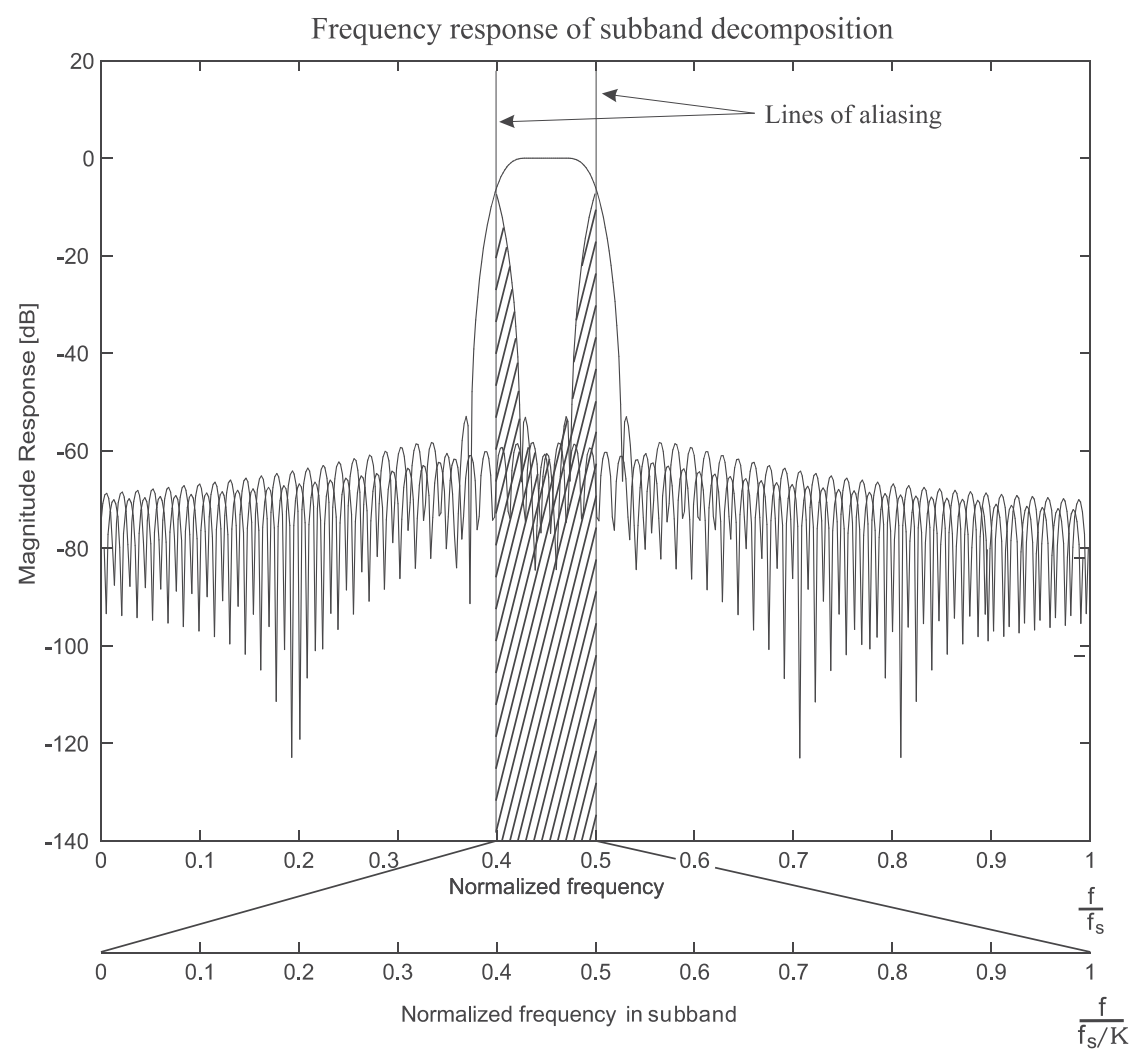

Fig. 3. The cause of the aliasing effect in critical sampling. 


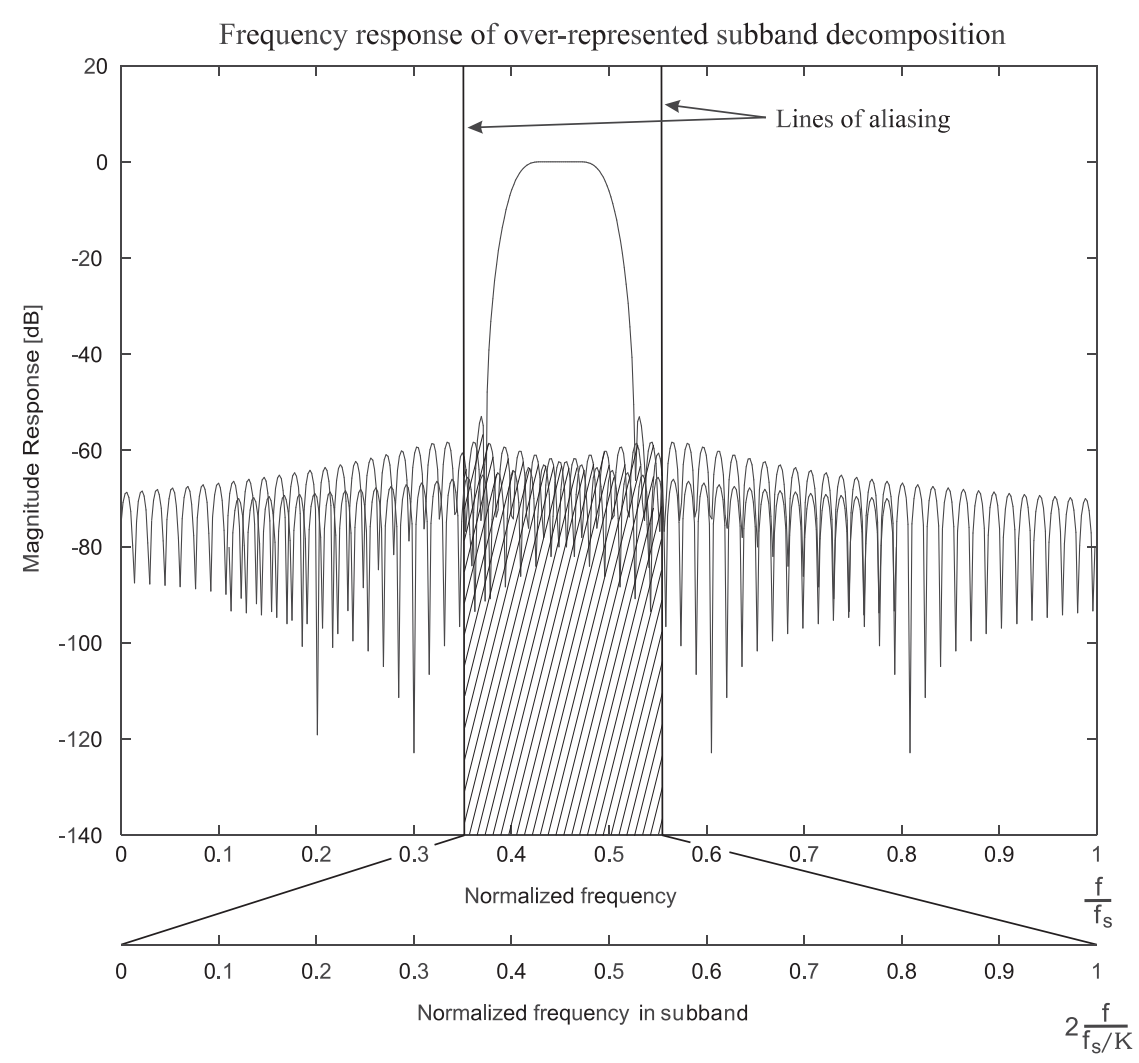

Fig. 4. The aliasing effect is reduced after over-sampling (2x).

\section{Prototype filter design}

A typical nonrecursive causal prototype filter can be defined by the transfer function

$H(z)=\sum_{n=0}^{L-1} h(n) z^{-n}$.

There are several ways to design this type of filter. One method is to use a window function. The filter coefficients $h(n)$ is given by the Window method as $h(n)=\frac{\sin \left(2 \pi f_{c}(n-(L-1) / 2)\right)}{\pi(n-(L-1) / 2)} w(n)$,

where $w(n)$ is a window function. For a given number of subbands, $\mathrm{M}$, and a given decimation/interpolation factor, D, and for a certain length of the prototype filter, L, we need to design the cut-off frequency $0<f_{c}<\frac{1}{2}$ and the corresponding window function.

A simple popular window function is the Hamming window, defined as

$w(n)=\left\{\begin{array}{lc}\alpha-(1-\alpha) \cos \frac{2 \pi n}{L-1} & \text { for } n=0,1, \ldots, L-1, \\ 0 & \text { otherwise }\end{array}\right.$ where $\alpha=0.54$. There is no additional parameter for this window function.

In case of the Kaiser window, there is an additional design parameter $\alpha$. Also, it can control the ripple ratio and the main-lobe width. This window is given by

$$
w(n)=\frac{\mathbf{I}_{0}\left(\alpha \sqrt{\left(\frac{L-1}{2}\right)^{2}-\left(n-\frac{L-1}{2}\right)^{2}}\right)}{\mathbf{I}_{o}\left(\alpha \sqrt{\left(\frac{L-1}{2}\right)^{2}}\right)},
$$

where $\mathbf{I}_{o}(x)$ is the zeroth-order modified Bessel function of the first kind.

Another window which can vary the ripple ratio and main-lobe width is the Dolph-Chebyshev window defined as

$w(n)=\frac{1}{L}\left[\frac{1}{r}+2 \sum_{i=1}^{(L-1) / 2} T_{L-1}\left(x_{0} \cos \mathrm{i} \pi / L\right) \cos (2 n \pi \mathrm{i} / L)\right]$,

where $r$ is the ripple ratio as a fraction, and

$x_{0}=\cosh \left(\frac{1}{L-1} \cosh ^{-1} \frac{1}{r}\right)$. 
Function $T_{k}(x)$ is the $k$ th-order Chebyshev polynomial assoicated with the Chebyshev approximation for recursive filters and is given by

$$
T_{k}(x)= \begin{cases}\cos \left(k \cos ^{-1} x\right) & \text { for }|x| \leqslant 1 \\ \cosh \left(k \cosh ^{-1} x\right) & \text { for }|x|>1\end{cases}
$$

The additional design parameter is therefore $r$ for this window.

Apart from using the window method, it is also possible to use the minimax technique instead. Once the cut-off frequency $f_{\mathrm{c}}$ and the stop-band frequency $f_{\mathrm{s}}$ is fixed, the minimax optimization problem can be solved quickly via the Remez exchange algorithm. Therefore, the two design parameters for the minimax method are $f_{\mathrm{c}}$ and $f_{\mathrm{s}}$.

In order to control the aliasing effect and the amplitude distortion separately, the final optimal design problem can be formulated into an equivalent optimization problem $(P 1)$ as

$\min _{\gamma} A_{P}(\boldsymbol{h}(\gamma))$

subject to

$A_{D}(\boldsymbol{h}(\gamma)) \leqslant a_{d}$,

where $a_{d}$ is a pre-defined tolerance for the amplitude distortion. The level of distortion can now be controlled freely. Note that $\gamma$ is one-dimensional for the Hamming window and two-dimensional for the other windows and the minimax method. $(P 1)$ is a nonsmooth constrained optimization problem. In order to simplify the problem, the $L_{1}$ exact penalty function is applied to convert it into an equivalent unconstrained minimization problem $(P 2)$ as

$\min _{\gamma} f(\gamma) \equiv A_{P}(\boldsymbol{h}(\gamma))+M \max \left(A_{D}(\boldsymbol{h}(\gamma))-a_{d}, 0\right)$,

where $M$ is a large number. In this way, constraint violations are penalized by a weighted $L_{1}$ term.

\section{Algorithms}

Since $(P 2)$ is a non-differentiable and highly nonlinear minimization problem, gradient-based approaches cannot adequately be used. Because the dimension of the problem is relatively low, a more realistic approach is to apply the simplex search technique to locate the minima via systematic moves in the solution space [8]. However, this still does not guarantee to reach the global minimum because of the nonlinearity. Consequently, a hybrid technique comprises the simulated annealing algorithm and the simplex search technique is proposed.

Theoretically, the global minimum of $f(\gamma)$ could be sought by using the simulated annealing algorithm alone. However, its convergence rate is usually very slow. On the other hand, the simplex search algorithm is much more efficient in converging to a stationary point. Thus, by combining the simulated annealing algorithm with the simplex search algorithm, we obtain an efficient hybrid descent method, which is formally stated in the following:

\section{A Hybrid Descent Algorithm}

1. Generate $\gamma^{(0)}$ randomly and evaluate $f\left(\gamma^{(0)}\right)$. Set $k=0$.

2. Solve for the local minimum of $f(\gamma)$ via the simplex search method with $\gamma^{(k)}$ as the initial guess to give $\gamma^{(k) \prime}$ such that $f\left(\gamma^{(k) \prime}\right)-f\left(\gamma^{(k)}\right) \leqslant 0$.

3. Starting from $\gamma^{(k) \prime}$, execute $\mathscr{N}$ simulating annealing iterations until a point $\gamma^{(k+1)}$ is obtained, such that $f\left(\gamma^{(k+1)}\right)-f\left(\gamma^{(k) \prime}\right) \leqslant 0$.

4. Set $k:=k+1$. Return to Step 2 until convergence.

In Step 3 of the algorithm, the simulated annealing iterations composes of three key steps, namely the generation of the next trial point in the solution space via random perturbations, a choice of a probability distribution to govern the acceptance of uphill steps, and an annealing schedule.

In this paper, following $[9,10]$, the Boltzmann probability distribution is used. The annealing schedule is determined by the parameters $\beta$, the cooling speed; $N_{\mathrm{c}}$, the number of cooling steps; $N$, the number of random perturbations for each temperature; and the initial temperature, $T$. Typical choices of these parameters can be found in [11]. The simulated algorithm algorithm can be implemented as follows:

Initiation: Select $\beta, N_{\mathrm{c}}, N$, and initial $T$. Evaluate $f\left(\gamma^{(k) \prime}\right)$.

Cooling

(a) Let $j$ be the cooling step. Set $j=1$.

(b) If $j \in\left(1, \ldots, N_{\mathrm{c}}\right)$

(i) $i=\operatorname{random}\{1,2\}$.

(ii) Depending on the outcome of $i$, re-generate randomly either one element of $\gamma$, or the whole vector of $\gamma$. This gives $\tilde{\gamma}$.

(iii) Calculate $D=f(\tilde{\gamma})-f(\gamma)$. If $D<0$ or $\operatorname{random}[0,1]<T \exp (-D / T)$, then $\gamma=\tilde{\gamma}$. 
(iv) Set $j:=j+1$ and return to (i) until $N$ perturbations are executed.

(c) Set $T:=\beta T$ and $j:=j+1$. Return to Step (b) until $N_{\mathrm{c}}$ cooling steps are executed.

\section{Evaluation results}

In the multi-criteria formulation, either the aliasing effect or the amplitude distortion can be imposed as a constraint. Since it is easier to assess the impact of the amplitude distortion for the application, it is therefore better to treat it as a constraint.

The first example is to assess different design of prototype filter and its implication to the aliasing effect for different oversampling factors. The amplitude distortion $a_{d}$ is first restricted to be $-20 \mathrm{~dB}$ and the result is depicted in Fig. 5. It is observed that by increasing the oversampling factor, the minimized aliasing effect is decreasing. However, the decrease is very slow for the Hamming window and is diminishing for the Remez method. Only the Kaiser and Dolph-Chebyshev window maintains a fast decrease in the minimized aliasing effect with the increase of the oversampling factor.
In this way, the Kaiser and Dolph-Chebyshev window out-performed other methods for all oversampling factor. We then varied the amplitude distortion $a_{d}$ requirement to $-30 \mathrm{~dB}$ and re-optimized the aliasing effect for different oversampling factors. The result is shown in Fig. 6. We observe a very similar pattern of performance. Also, we observe a trade-off between the amplitude distortion and the optimized aliasing effect, which is expected due to the multi-criteria nature of the problem. The choice of $a_{d}$ depends largely on the application requirements.

In order to understand the influence of the prototype filter length and the number of subbands, we apply the Kaiser window as a demonstration. As the number of subbands increases, the passband gets narrower. Thus, it is hard to maintain the low distortion level unless the length of the filter increases to allow for a narrower transition region. If the filter length is proportional to the number of subbands, the result is shown in Fig. 7. It is interesting to see that as the number of subbands increases, there is a uniform improvement in the minimized aliasing effect for all oversampling levels. The improvement is more significant for sampling factors near to

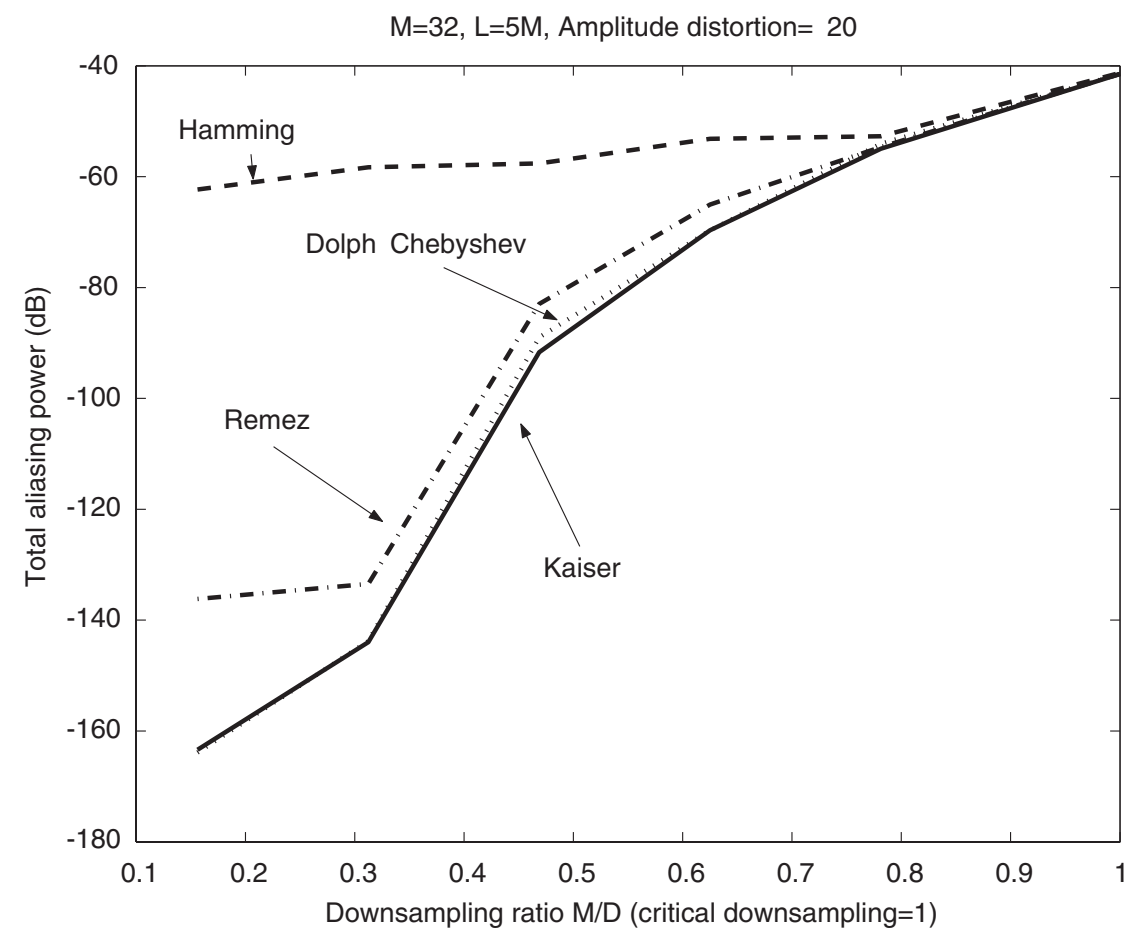

Fig. 5. Comparison for different design methods. 


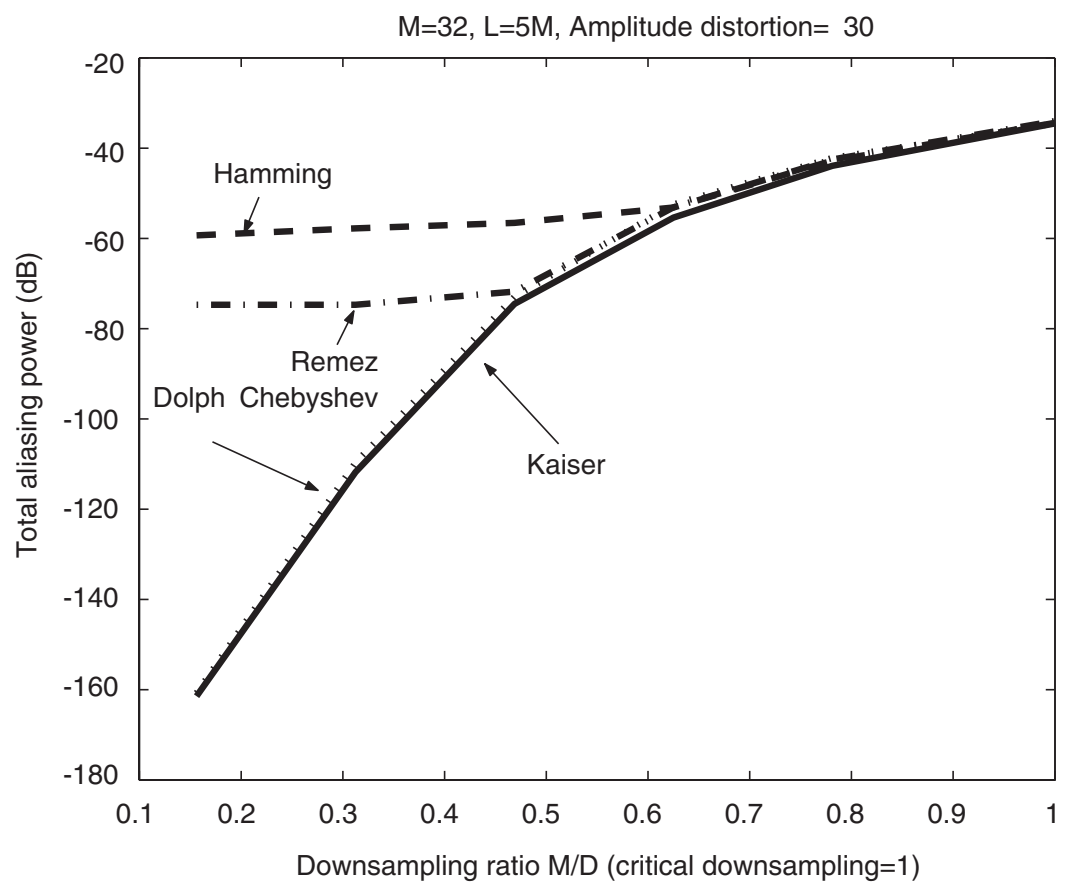

Fig. 6. Comparison for different design methods.

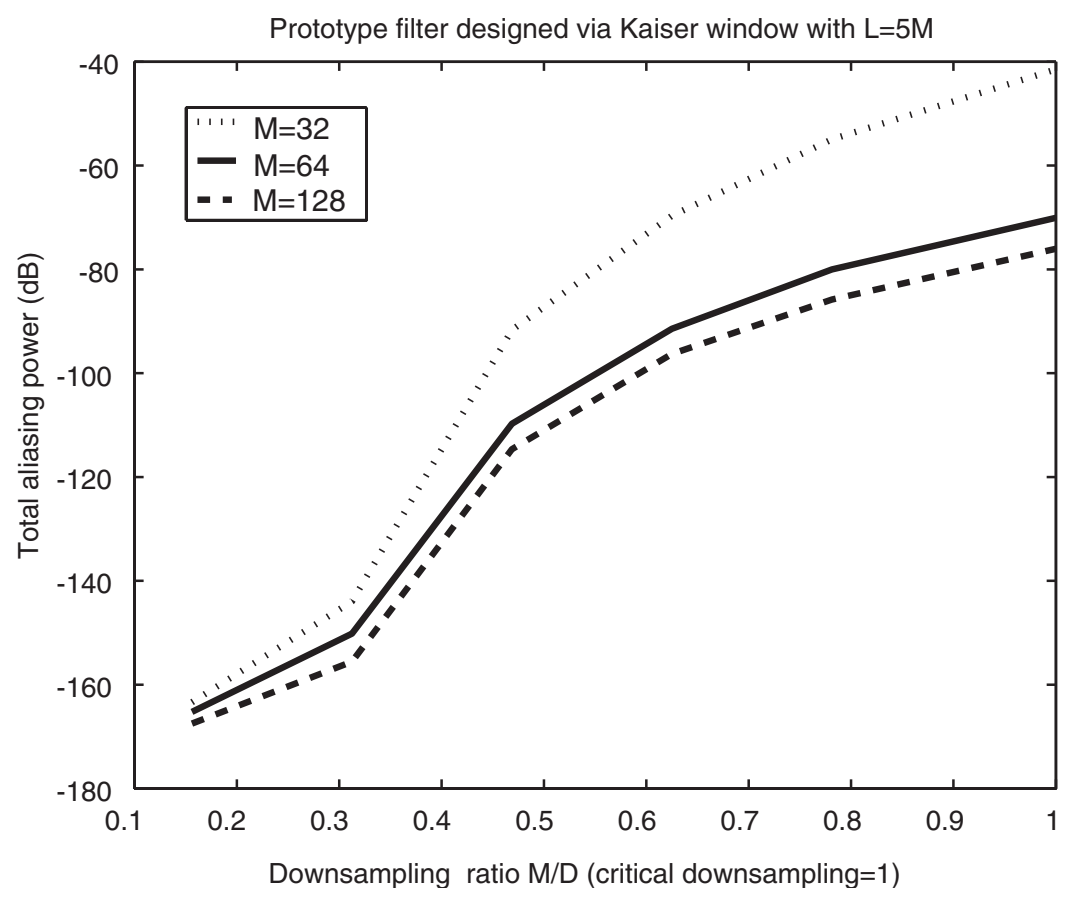

Fig. 7. Comparison for different number of subbands.

critical one. The final designs of prototype filters are shown in Fig. 8. All designs have a similar level of minimized aliasing effect; however, they all have different transition widths and stopband ripples. As the maximum of the aliasing effect usually occurs near to the transition region, the 
$M=32, D=32, L=5 M$, amplitude distortion $=-20$

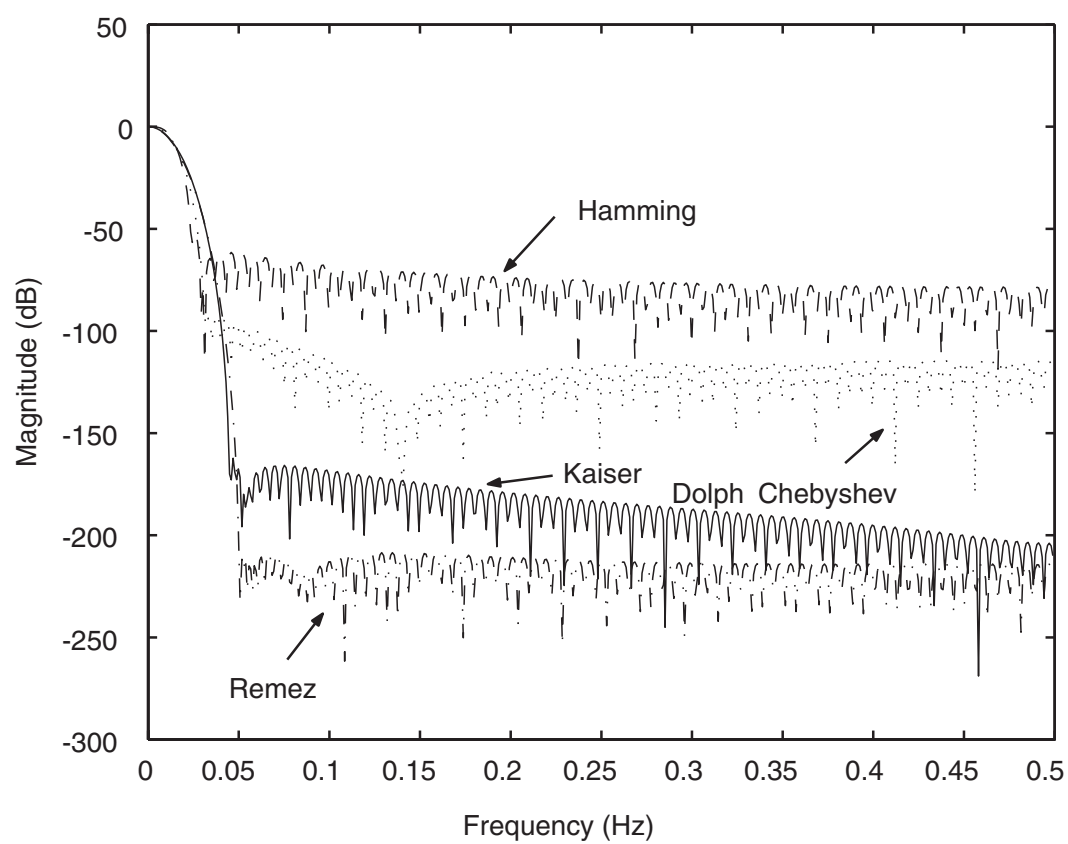

Fig. 8. Comparison for different designs with critical sampling.

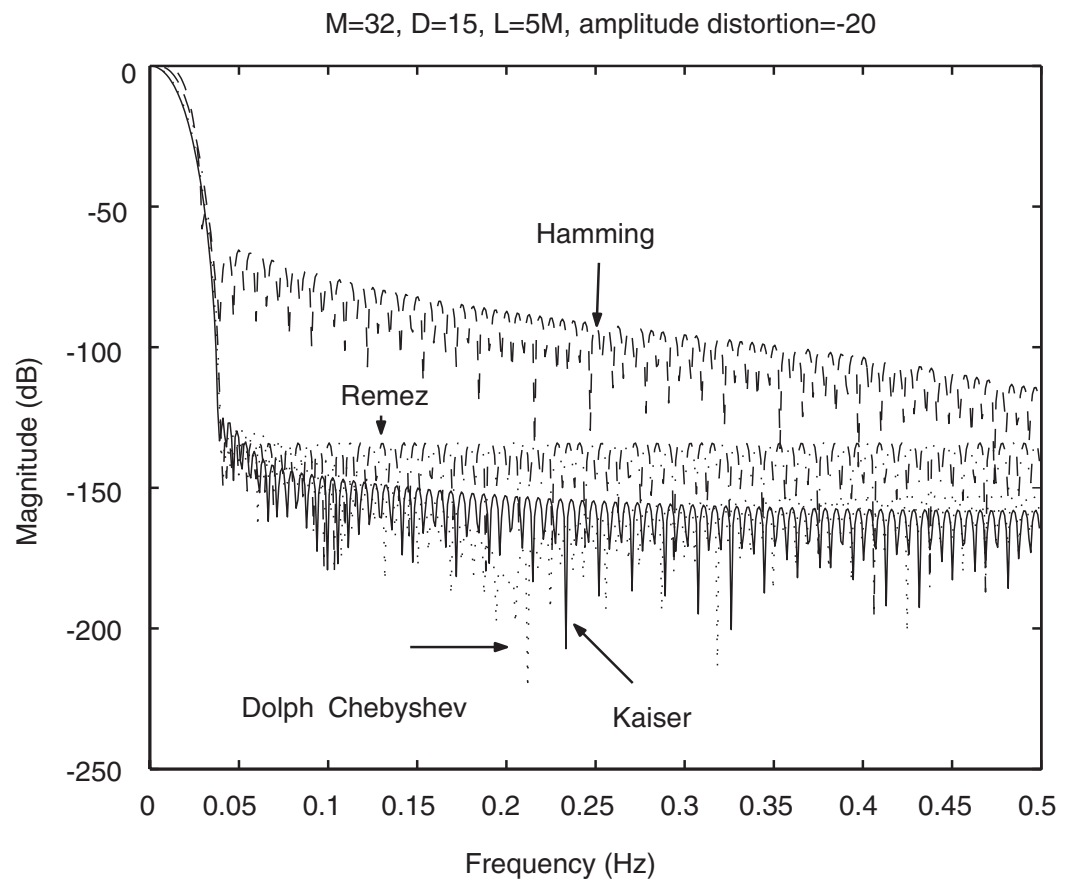

Fig. 9. Comparison for different designs with over-sampling.

transition width and the stopband ripple clearly compensates for each other. When oversampling factor about 0.5 is applied, the result is given in
Fig. 9. From the figure, the transition widths are very similar for different designs. However, there are significant differences in the stopband ripples. 
This account for the differences in the aliasing effect suppression.

\section{Conclusions}

In this paper, the problem of filter bank design has been studied. The design problem has been formulated as a multi-criteria optimization problem. Using the $L_{1}$ exact penalty function, a new hybrid method is proposed to tackle the problem. Consequently, all the Pareto optima can be solved and studied. Different designs of the prototype filter have been investigated. It turns out that Kaiser and Dolph-Chebyshev window give the best performance with the lowest aliasing effect for a fixed amplitude distortion level. If the length of the prototype filter is proportional to the number of subbands, the optimal aliasing effect generally improves with the number of subbands. The improvement is more significant for sampling factors close to the critical one.

\section{Acknowledgements}

The third author would like to thank ARC, the Australian Research Council for supporting this research under grant DP0451111. The fourth author would also like to thank ARC for supporting this research.

\section{References}

[1] P.P. Vaidyanathan, Multirate Systems and Filter Banks, Prentice-Hall, Englewood Cliffs, NJ, 1993.

[2] S. Haykin, Adaptive Filter Theory, Prentice-Hall, Englewood Cliffs, NJ, 2002

[3] A. Gilloire, M. Vetterli, Adaptive filtering in sub-bands with critical sampling: analysis, experiments, and application to acoustic echo cancellation, IEEE Trans. Signal Process. 40 (8) (1992) 1862-1875.

[4] W. Kellermann, Analysis and design of multirate systems for cancellation of acoustic echoes, in: Proceedings of ICASSP, 1988, pp. $2570-2573$.

[5] J.M. de Haan, N. Grbić, I. Claesson, S. Nordholm, Design of oversampled uniform DFT filter banks with delay specification using quadratic optimization, in: Proceedings of ICASSP, 2001.

[6] M. Zeleny, Multiple Criteria Decision Making, McGrawHill, New York, 1982.

[7] T.L. Vincent, W.J. Grantham, Optimality in Parametric Systems, Wiley, New York, 1981.

[8] R. Fletcher, Practical Methods of Optimization, Wiley, New York, 1987.

[9] S. Kirkpatrick, C.D. Gelatt Jr., M.P. Vecchi, Optimization by simulated annealing, Science 220 (1983) 671-680.

[10] V. Cerny, Thermodynamical approach to the traveling salesman problem: an efficient simulation algorithm, J. Optim. Theory Appl. 45 (1985) 41-51.

[11] W.H. Press, S.A. Teukolsky, W.T. Vetterling, B.P. Flannery, Numerical Recipes in Fortran: The Art of Scientific Computing, second ed., Cambridge University Press, Cambridge, 1992. 